Z. Physik 267, 410 (1974)

(C) by Springer-Verlag 1974

\title{
Erratum
}

\section{Decay of ${ }^{51} \mathrm{Mn}$}

J.C. Ferrer, J. Rapaport, and S. Raman

Z. Physik 265, 365-370 (1973)

In the table appearing in the above article, the uncertainty in the energy of the $2001.35 \mathrm{keV} \gamma$-ray should be 0.12 instead of 1.12 .

J. Rapaport

Physics Department

Ohio University

Athens, Ohio 45701

USA 